\title{
Disrupted Working Memory Circuitry in Schizophrenia: Disentangling fMRI Markers of Core Pathology vs Other Aspects of Impaired Performance
}

\author{
Hamdi Eryilmaz", , Alexandra S Tanner', New Fei Ho', Adam Z Nitenson', Noah J Silverstein', \\ Liana J Petruzzi', Donald C Goff', Dara S Manoach' and Joshua L Roffman' \\ 'Department of Psychiatry, Massachusetts General Hospital and Harvard Medical School, Charlestown, MA, USA; ${ }^{2}$ Department of Psychiatry, \\ New York University Langone Medical Center and Nathan Kline Institute, New York, NY, USA
}

\begin{abstract}
Working memory (WM) impairment, a core feature of schizophrenia, is often associated with aberrant dorsolateral prefrontal cortex (dIPFC) activation. Reduced resting-state connectivity within the frontoparietal control network (FPCN) has also been reported in schizophrenia. However, interpretation of WM-related dIPFC dysfunction has been limited by performance differences between patients and controls, and by uncertainty over the relevance of resting-state connectivity to network engagement during task. We contrasted brain activation in 40 schizophrenia patients and 40 controls during verbal WM performance, and evaluated underlying functional connectivity during rest and task. During correct trials, patients demonstrated normal FPCN activation, despite an inverse relationship between positive symptoms and activation. FPCN activation differed between the groups only during error trials (controls $>$ patients). In contrast, controls demonstrated stronger deactivation of the ventromedial prefrontal cortex (vmPFC) during correct and error trials. Functional connectivity analysis indicated impaired resting-state FPCN connectivity in patients, but normal connectivity during task. However, patients showed abnormal connectivity among regions such as vmPFC, lateral orbitofrontal cortex, and parahippocampal gyrus (PHG) during both rest and task. During task, patients also exhibited altered thalamic connectivity to PHG and FPCN. Activation and connectivity patterns that were more characteristic of controls generally correlated with better performance. In summary, patients demonstrated normal FPCN activation when they remained on-task, and exhibited normal FPCN connectivity during WM, whereas vmPFC deactivation differences persisted regardless of WM performance. Our findings suggest that altered FPCN activation in patients reflects performance difference, and that limbic and thalamic dysfunction is critically involved in WM deficits in schizophrenia.
\end{abstract}

Neuropsychopharmacology (20 I6) 4I, 24I I-2420; doi: I 0. I038/npp.20 I6.55; published online I I May 2016

\section{INTRODUCTION}

Working memory (WM) is an important component of higher cognition and is essential for goal-directed behavior (D'Esposito, 2007). Impaired WM is a well-documented symptom in schizophrenia and arguably a core feature of the disease. Many studies investigating WM deficits in schizophrenia focus on the dorsolateral prefrontal cortex (dlPFC), a region implicated in executive functions, goal-directed planning, and inhibition (Jansma et al, 2004; Manoach et al, 2000; Potkin et al, 2009). However, the direction of dlPFC findings has been inconsistent across studies, which have demonstrated both diminished and enhanced dlPFC activation in schizophrenia (Carter et al, 1998; Manoach et al, 1999; Potkin et al, 2009).

\footnotetext{
*Correspondence: Dr H Eryilmaz, Department of Psychiatry, Massachusetts General Hospital and Harvard Medical School, 149 13th Street, Room 2606, Charlestown, MA 02129, USA, Tel: + I 617643 7462, Fax: + 617726 4078, E-mail: hamdi.eryilmaz@mgh.harvard.edu Received 15 October 2015; revised 3 March 2016; accepted 7 April 2016; accepted article preview online 22 April 2016
}

Several factors could contribute to this discrepancy (Manoach, 2003). Schizophrenia patients frequently perform worse than controls in WM tasks (Johnson et al, 2006; Meda et al, 2009), confounding brain activation differences when the groups are compared at the same WM load. This is especially problematic in block designs that average across correct and error trials. Alternatively, the groups are sometimes compared at different task loads to match performance (Johnson et al, 2006). However, this approach is also problematic as the tasks are not identical for both groups.

Without fully controlling for performance differences over the same dynamic range of WM load, it is difficult to know whether performance deficits in schizophrenia reflect primary dysfunction in task-related networks, suboptimal activation of these networks, and/or downstream effects of other disrupted networks (Van Snellenberg et al, 2006). Indeed, WM function depends not only on activation of dlPFC and other nodes within the frontoparietal control network (FPCN) but also on deactivation of the default mode network (DMN; Anticevic et al, 2010). Numerous recent 
Table I Demographics, Clinical Measures, and Working Memory Task Performance

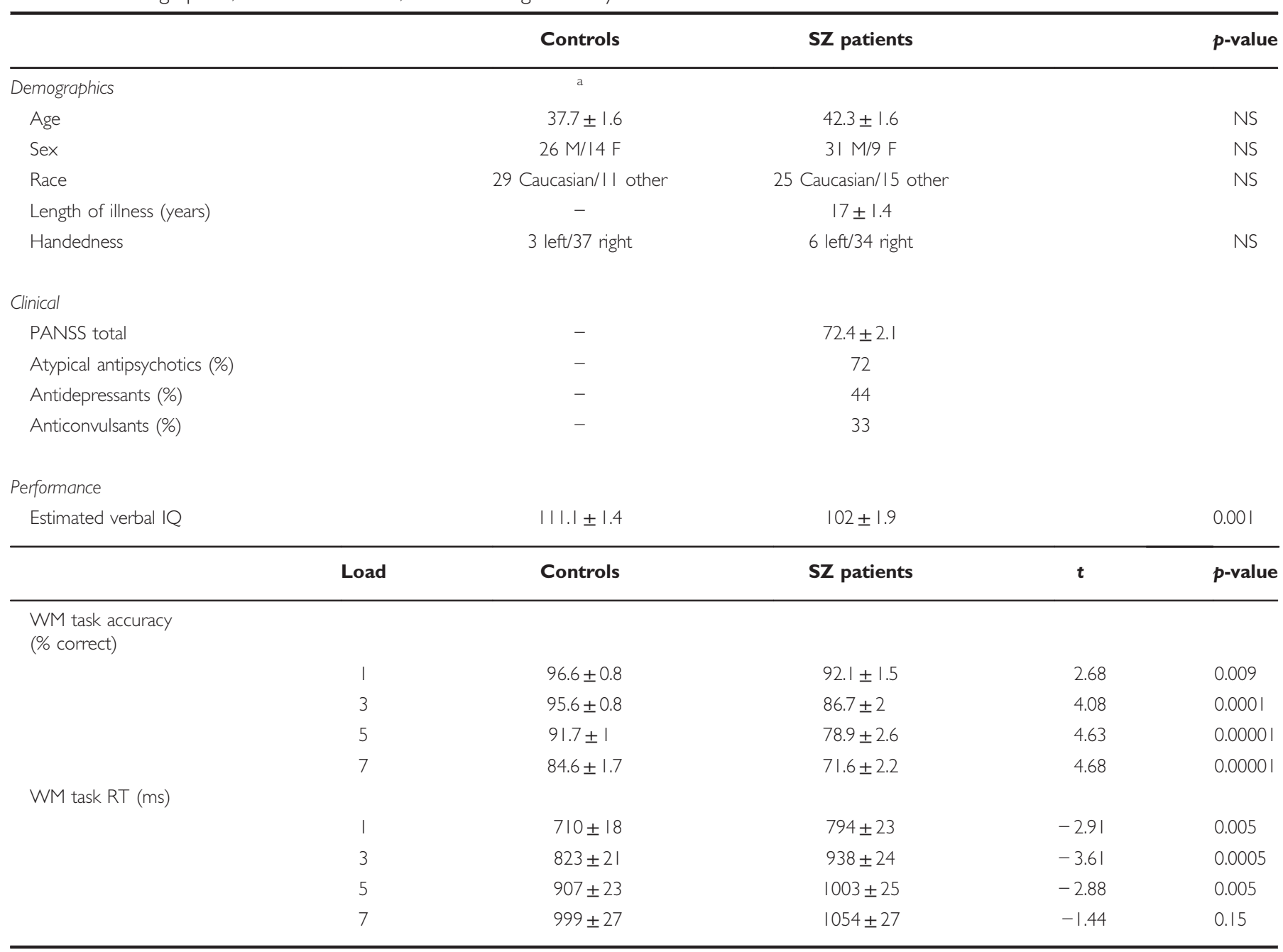

Abbreviations: F, female; M, male; NS, not significant; PANSS, Positive and Negative Syndrome Scale; RT, reaction time; SZ, schizophrenia; WM, working memory. ${ }^{\mathrm{a}}$ Mean $\pm \mathrm{SEM}$ is shown for quantitative parameters.

studies have identified altered DMN activation and connectivity in schizophrenia (Kim et al, 2009; Meda et al, 2009), complementing a large volume of work implicating FPCN dysfunction (Karlsgodt et al, 2007; Manoach et al, 2000; Minzenberg et al, 2009; Potkin et al, 2009). However, the relationship between WM deficits and both task-related activation and intrinsic network connectivity remains incompletely understood.

We used an event-related version of the Sternberg Item Recognition Paradigm (SIRP) to investigate WM in schizophrenia patients and controls. Unlike other WM paradigms such as the Wisconsin Card Sorting Test and n-back, the SIRP focuses primarily on the maintenance of information, and the task is associated with linear changes in response time, activation of FPCN, and deactivation of DMN, as a function of WM load (Kim et al, 2009; Sternberg, 1966; Yendiki et al, 2010). An event-related design allowed us to separately examine load-dependent activation associated with correct $v s$ error trials. For regions that differed between patients and controls, we used resting and task-based functional connectivity analyses to better understand differences in network dynamics that lead to group differences during WM performance.

\section{MATERIALS AND METHODS}

\section{Participants}

Chronic, medicated outpatients with schizophrenia $(n=40)$, and age- and gender-matched healthy controls $(n=40)$ participated in this fMRI study. Exclusion criteria included history of significant head injury, substance abuse, or neurological disease. Patients were evaluated with the Positive and Negative Syndrome Scale (PANSS) and Scale for Assessment of Negative Symptoms (SANS). Demographics, medication use, and symptom severity are listed in Table 1. All participants gave written informed consent. The protocol was approved by the Partners HealthCare Human Research Committee. 


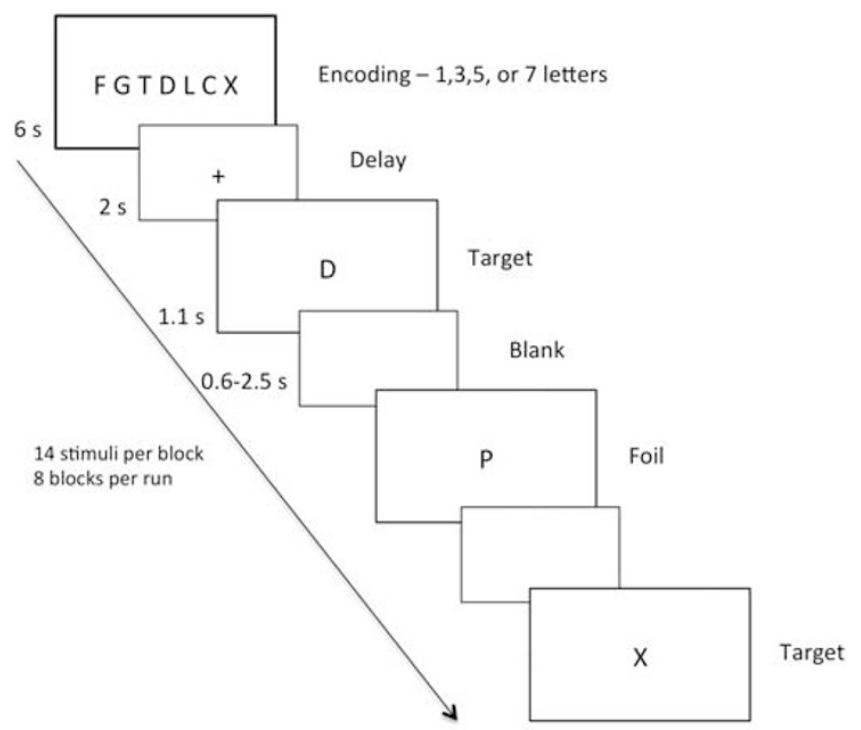

Figure I Illustration of Sternberg Item Recognition Paradigm (SIRP). Each block started with presentation of the memorized set, which consisted of I, 3, 5, or 7 letters. Then, single letters were presented in succession. Subjects' task was to press 'I' on the keypad if the letter was a foil (not a letter in the memorized set), and to press ' 2 ' if it was a target. Subjects completed three 7 -min runs in the scanner, each of which consisted of 112 trials.

\section{Task}

The SIRP was used to evaluate WM function at four different loads (1, 3, 5, and 7 letters). Before scanning, subjects performed a practice run to ensure that they understood the task. To ensure adequate task engagement, subjects were only included in the primary analysis if they demonstrated abovechance performance during the practice run, and during (at least) the easiest load condition during the scan. EPRIME 1.1 software presented the task during scans and collected participants' responses. Each block of the task comprised encoding, delay, and multiple probe epochs (Figure 1). The encoding phase started with presentation of a set of $1,3,5$, or 7 consonants $(6 \mathrm{~s})$. Then, the delay epoch was introduced with a fixation cross $(2 \mathrm{~s})$, followed by presentation of 14 probes (consonants) for $1.1 \mathrm{~s}$, separated by a varying intertrial interval. Participants indicated, using a keypad placed in the dominant hand, whether the letter displayed on the screen was a target (ie, presented during the encoding phase; $50 \%$ of probes) or a foil (not presented during encoding; 50\% of probes). Probes were jittered (range 1.7-3.6 s) using OptSeq to facilitate event-related analysis (http://surfer.nmr.mgh.harvard.edu/optseq/). Each of the 4 task loads was used twice in a single run, totaling 112 trials and 8 blocks. Participants completed three task runs that lasted $7 \mathrm{~min} 20 \mathrm{~s}$ each.

\section{MRI Acquisition and Preprocessing}

MRI data were collected in a 3T Siemens TIM Trio System with a 12-channel quadrature head coil. Preprocessing, motion correction, and general linear model (GLM) analyses were conducted using FreeSurfer Functional Analysis Stream software (Burock and Dale, 2000). In addition to standard motion correction, we censored time points and removed runs that exceeded predetermined motion thresholds (see Supplementary Materials and Methods for additional details).

\section{GLM Analysis}

We analyzed activations in the probe epochs, during which subjects mentally scanned the memorized set, decided whether the probe letter belonged to the set, and executed a motor response. In our GLM design, each epoch began when the probe stimulus was presented and continued until the following stimulus was presented. The design matrix included two main regressors of interest: correct response and incorrect response. Each probe trial was assigned a load coefficient $(1,3,5$, or 7$)$ depending on the number of letters to be memorized. For all task-based fMRI activation analyses, we used a standard GLM to evaluate whether task load (number of letters to hold online) was linearly related to activation at each voxel (for volume-based analyses) or vertex (for surface-based analyses). Epochs were convolved with a gamma hemodynamic response function (delay $=2.25 \mathrm{~s}$, dispersion $=1.25 \mathrm{~s}$; Dale and Buckner, 1997). Using an eventrelated parametric design allowed us to analyze activations arising during correct and error trials separately. Encoding periods were also included as regressors. Skipped responses were considered errors. The contrasts 'Correct $v s$ Fixation' and 'Error vs Fixation' examined WM load-dependent activation arising in response to correct and error trials, respectively. Once parameter estimates were computed for each subject, they were entered into a second-level random-effects group analysis to compute group average and group difference maps, which were then correlated with accuracy and symptom severity in second-level analyses. For additional details see Supplementary Materials and Methods.

For all GLM analyses, we used 10000 Monte Carlo simulations to correct for multiple comparisons across the surface or volume (Hagler et al, 2006). Before simulations all maps were thresholded using a height threshold (set to $p<0.005$ ). Monte Carlo analysis then determined the likelihood that resulting clusters with a certain size would be found by chance (cluster-wise probability). The corrected $p$-value was set to 0.05 (two-tailed).

\section{Functional Connectivity Analysis}

To better understand the mechanisms underlying group activation differences, we analyzed functional connectivity during both resting and task states. Seeds were created using a 6-mm sphere around the peak of activation difference between the groups (Supplementary Table S1). For each seed region, functional connectivity maps were generated by computing the correlation between the mean signal time course from this seed and the time courses from all voxels using Pearson's product moment correlation. For task-based connectivity, correlation values were averaged across the three SIRP runs to generate a single task connectivity map per subject. These maps were then converted to z-maps using Fisher's $\mathrm{z}$ transformation, which enhances the normality of the distribution of correlations. One-way ANOVA was then used for each voxel to test for group differences. We determined significance at cluster-wise $p<0.05$ corrected for 

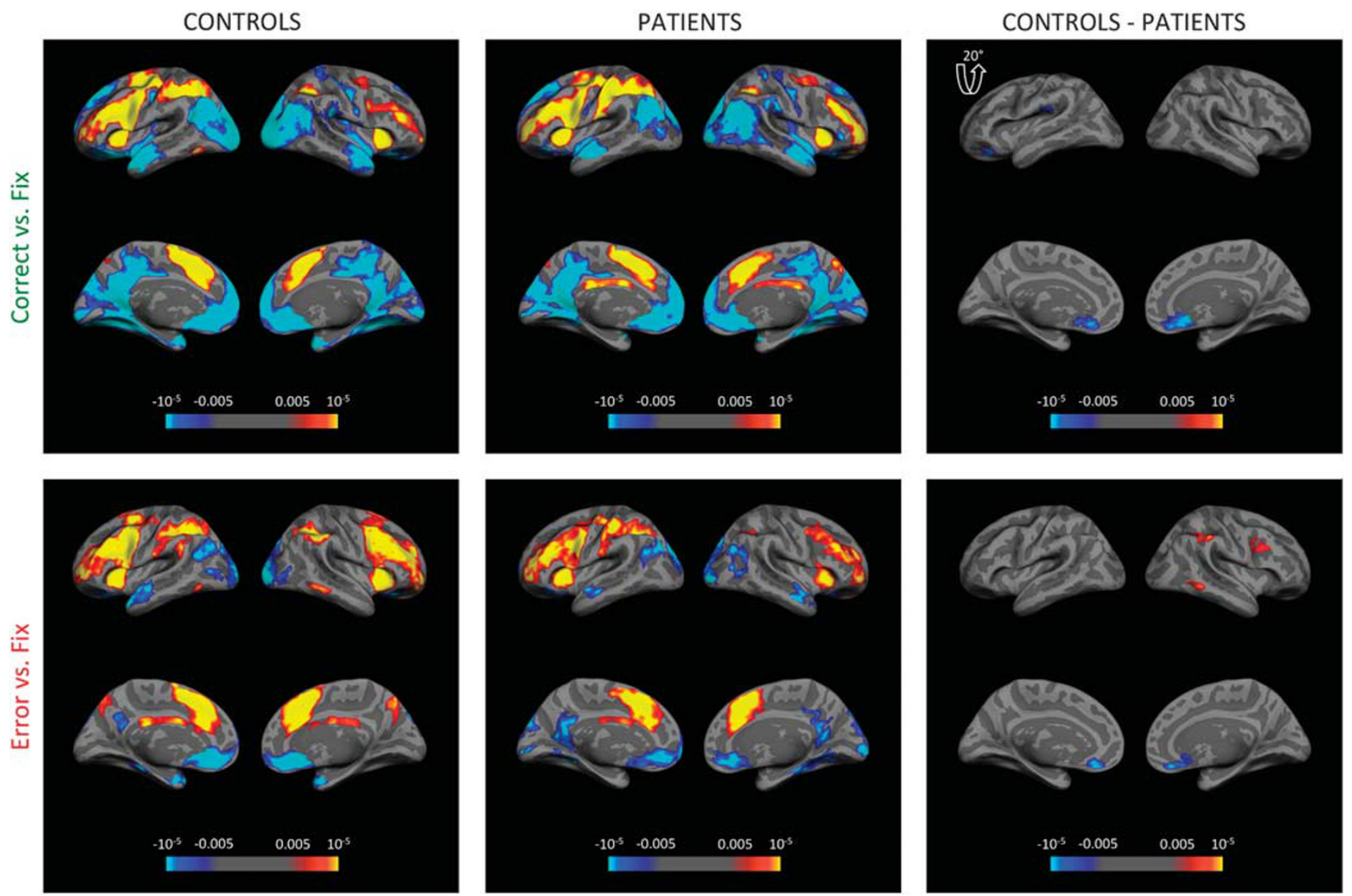

Figure 2 Brain regions showing parametric activation in response to increasing task load during correct and error epochs. Each surface map is represented by four images with lateral and medial views on the left and right hemisphere. Maps for both control (left) and patient (middle) groups are shown together with group difference maps (right). Left lateral view of the group difference map for Correct vs Fix was tilted $20^{\circ}$ for a better view of the postcentral gyrus. Both controls and patients demonstrated load-dependent activation in FPCN and deactivation in the DMN during correct epochs. Deactivation in mPFC was stronger in controls during both conditions. During error epochs, activation in the right dIPFC, SMG, and MTG was selectively stronger in controls. Colorbar denotes the $p$-value. All clusters displayed on maps survive correction for multiple comparisons using 10000 Monte Carlo simulations at clusterwise $p<0.05$.

multiple comparisons using a combination of uncorrected height threshold $(p<0.0001)$ and a minimum cluster size, which was determined using AlphaSim with the following input parameters: Gaussian filter FWHM $=7.2 \mathrm{~mm}$, cluster connection radius $=2 \mathrm{~mm}$, number of Monte Carlo simulations $=10000$, and voxel threshold probability $=0.001$. The mask was set to the whole-brain template. FWHM was calculated by averaging individual smoothness values that were estimated on subjects' residuals from a linear regression analysis, in which time course of each seed was regressed out from the preprocessed data. We used a more conservative height threshold for the connectivity analysis, as it is a more indirect measure and is arguably more sensitive to confounding sources than task-based activation, and therefore might be more prone to type 1 errors (Buckner, 2010). Our pipeline also took sign of correlations into account. We reported pairs of regions for which the correlation was either positive in both the groups or positive in one group and its absolute value was smaller than 0.1 in the other, as it is remains unclear that negative time-course correlations can be interpreted as meaningful (Chai et al, 2012; Scholvinck et al, 2010). In addition to the standard functional connectivity analysis described above, we performed a correlation analysis to assess whether the strength of connectivity between regions that showed significant between-group differences is associated with behavioral measures such as accuracy and symptom severity. For this, we computed the correlation between connectivity of all pairs that showed significant between-group differences and task accuracy (across both groups) and symptom severity (in the patient group). We used Bonferroni correction to control for multiple comparisons across pairs $\left(p_{\text {corrected }}<0.05\right.$; $\left.p_{\text {uncorrected }}<0.00167\right)$.

\section{RESULTS}

\section{Behavioral Results}

All included participants showed overall accuracy performance that was significantly above chance in at least one of the task loads. Controls performed more accurately than patients at all load levels, and responded more quickly at loads 1,3 , and 5 (Table 1). 


\section{GLM Results}

Between-group differences in load-dependent activation during correct and error epochs ( $v s$ fixation periods) are shown in Figure 2 and Supplementary Table S1.

Correct epochs. Both controls and patients showed robust load-dependent FPCN activation in correct epochs, and notably this activation did not differ between the groups. This pattern was also evident when separately examining each load (Supplementary Figure S1). However, we observed stronger deactivation in the left lateral orbitofrontal cortex (lOFC) and bilateral ventromedial prefrontal cortex (vmPFC) in controls compared with patients. Conversely, patients showed greater activation in left postcentral gyrus.

Error epochs. During error epochs, controls showed significantly greater activation in FPCN territory, including right supramarginal gyrus (SMG), middle temporal gyrus (MTG), and dIPFC (Figure 2). In addition, voxel-wise analysis revealed that left thalamus was more strongly activated in controls during error epochs (Supplementary Figure S2). Controls again showed significantly stronger deactivation in bilateral vmPFC.

Correct vs Error epochs. Within-group analysis contrasting correct and error trials revealed that right frontoparietal areas, dorsal anterior cingulate, and precuneus showed increased activation during error trials ( $v s$ correct) in controls (Supplementary Figure S3). Patients showed reduced activation in pre- and postcentral gyrus, but increased activation in bilateral supramarginal gyrus, precuneus, and superior frontal gyrus during error epochs ( $v s$ correct).

Performance correlations. Correlation with accuracy did not reach significance for any brain regions during correct trials. During error epochs, accuracy correlated positively with the degree of activation in regions of the FPCN, including bilateral superior parietal lobule (SPL), bilateral dlPFC, dorsomedial prefrontal cortex (dmPFC), and bilateral insula, and with the degree of deactivation in regions of the $\mathrm{DMN}$, including posterior cingulate cortex and medial prefrontal cortex (Supplementary Figure S4A). Post hoc analyses of significant regions revealed interactions of the group X activation on accuracy in six predominantly tasknegative areas; performance depended on activation more strongly among patients (Supplementary Figure S4B).

Symptom correlations. An inverse correlation was observed in patients between severity of PANSS positive subscale score and activation in left and right dlPFC during correct epochs (Supplementary Figure S5). No significant correlations were found for activity during error epochs or for SANS scores.

Additional analyses. See Supplementary Results and Supplementary Figures S6 and S7 for additional analyses concerning symptom-performance correlations, exclusion of below-chance performance at higher WM loads, and response time differences.

\section{Functional Connectivity}

Functional connectivity analyses were performed for both resting and task states. Seed regions were based on the primary task-based GLM analysis (Supplementary Table S1). To further control for head motion, an important potential confound in functional connectivity (Power et al, 2012), we conducted additional quality control analyses in which we removed problematic runs from the analysis using a stringent $(0.6 \mathrm{~mm})$ movement threshold; results were largely unchanged (Supplementary Results).

Resting-state connectivity. Supplementary Table S2 and Figure 3 display region pairs for which functional connectivity differed significantly between the groups at rest. In patients, two different seeds in left vmPFC, which had been associated with reduced task-based deactivation, also showed abnormally strong connectivity with $1 \mathrm{OFC}$, another region in the cortical limbic network (as defined by Yeo et al, 2011). Connectivity of left postcentral gyrus with left insula, right inferior frontal gyrus, and left thalamus was also abnormally enhanced in patients.

Right dlPFC, a region parametrically activated with increasing task load, showed reduced coupling with right inferior temporal gyrus (ITG) in patients. In the right hemisphere, we also observed stronger connectivity between SMG and MTG in controls as compared to patients. These results overall indicate stronger coupling within the FPCN in controls and a shift in patients' connectivity patterns within the networks involved in sensory and affective processing.

Task-state connectivity. Supplementary Table S2 and Figure 4 depict brain regions that showed significantly different task connectivity between the groups. Coupling between vmPFC and right parahippocampal gyrus was stronger in controls. We also observed stronger coupling from right SMG to right pallidum and thalamus in controls. Left thalamus showed weaker coupling with bilateral dmPFC, right dACC, and left IPL (all part of FPCN) in patients, whereas its connectivity was enhanced with parahippocampal gyrus and Rolandic operculum in patients as compared with controls. Notably, however, connectivity within cortical FPCN nodes did not differ between patients and controls during task, as they did at rest.

Behavioral correlations. For each pair of regions that showed significant difference in functional connectivity between the two groups, we evaluated the correlation between connectivity of that pair and accuracy (across all participants) as well as between connectivity and PANSS/SANS scores (in patients). Bonferroni correction controlled for multiple comparisons across connectivity pairs. None of the pairs showed significant correlation with PANSS or SANS scores. Accuracy correlated positively with restingstate connectivity within the somatomotor network (between two locations in left postcentral gyrus, $p=0.0005, r=0.381$ ). Accuracy correlated negatively with resting-state coupling between left vmPFC and right lOFC (limbic network, $p=0.00045, r=-0.384)$, and between left postcentral gyrus and left insula $(p=0.00011, r=-.0419)$-both of which showed patient $>$ control connectivity. Accuracy correlated positively with task-state thalamocortical connectivity (left 


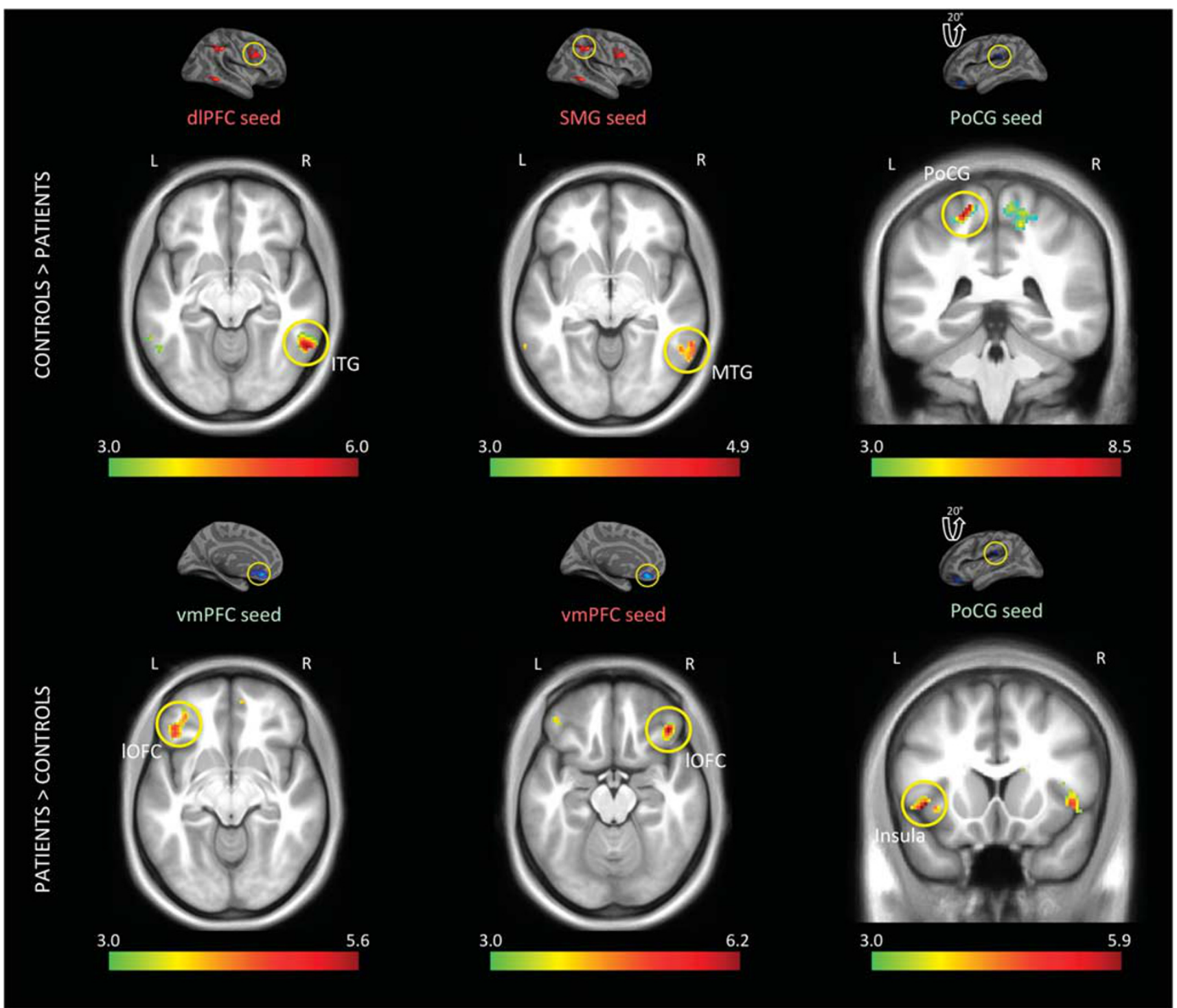

Figure 3 Differences in functional connectivity between the groups at rest. Seed regions are indicated with a circle on their respective activation maps above the connectivity maps, which reflect the p-values derived from an ANOVA. Colored seed labels represent the effect from which the seed was defined; green: Correct vs Fix, red: Error vs Fix. Right dIPFC was more strongly coupled with right ITG in controls, whereas left vmPFC was more strongly coupled with bilateral IOFC in patients. In controls, right SMG showed stronger coupling with right MTG. Controls also showed enhanced coupling within left postcentral gyrus, whereas patients showed increased coupling between this region and left insula. Colorbar denotes the $-\log (p)$ value and all the effects are shown at $p<0.00$ I for illustration purposes.

thalamus and right dmPFC, $p=0.00034$ and $r=0.391$ ), which showed control $>$ patient connectivity.

\section{DISCUSSION}

Although prefrontal dysfunction has been consistently implicated in fMRI studies of schizophrenia, differences in WM performance have posed a ubiquitous confound in interpreting activation differences between patients and controls (Minzenberg et al, 2009). Further, previous functional connectivity work has largely been limited to restingstate studies, leaving uncertain the relevance of resting-state FPCN abnormalities to its recruitment during WM. Here using event-related fMRI analysis in parallel with resting and task-based connectivity, we demonstrate that patients are capable of normal FPCN activation when they are adequately engaged in the task (as gauged by correct performance). Further, patient deficits in FPCN connectivity during rest, which were similar to previous studies (Baker et al, 2014), do not persist during WM performance. Conversely, patients consistently demonstrate impaired deactivation of vmPFC (a limbic 'task-negative' region) regardless of accuracy, as well as altered vmPFC and thalamic connectivity during WM. These findings challenge the primacy of frontoparietal dysfunction during WM in schizophrenia; alternately, they suggest that although dlPFC (and, more broadly, FPCN) dysfunction is a marker of performance differences, limbic and thalamic network disruptions are critically involved in WM deficits. 


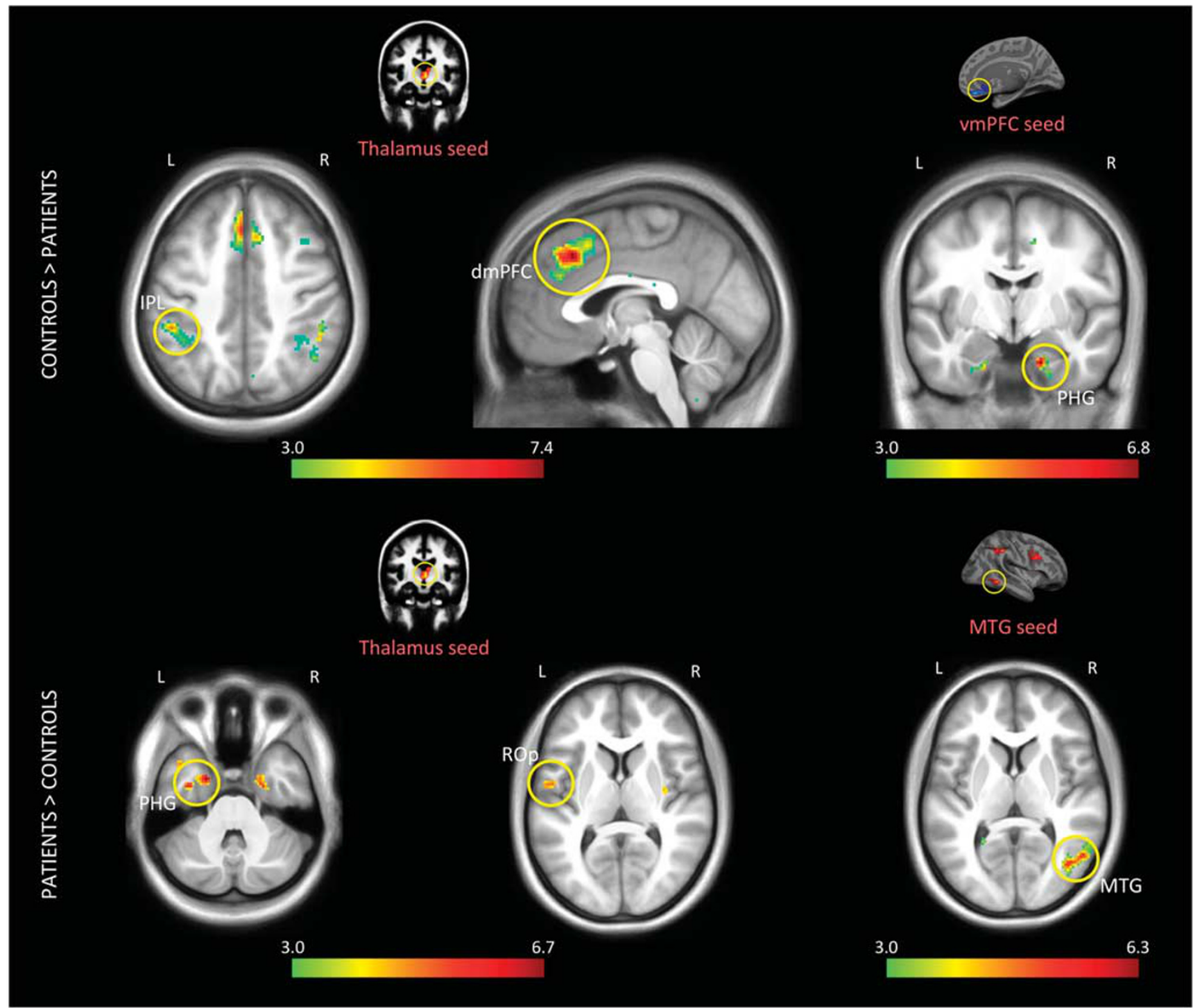

Figure 4 Differences in functional connectivity between the groups during the task. Seed regions are indicated on their respective activation maps above the connectivity maps, which reflect the p-values derived from an ANOVA based on the average of three task runs in each subject. Colored seed labels represent the effect from which the seed was defined; red: Error vs Fix. The left thalamus showed enhanced coupling with dmPFC and left IPL in controls, whereas its connectivity to left PHG and left ROp was enhanced in patients. Functional connectivity between vmPFC and right PHG was enhanced in controls. Schizophrenia patients also showed increased coupling between middle temporal regions. Colorbar denotes the $-\log (p)$ value and all the effects are shown at $p<0.00$ I for illustration purpose.

\section{FPCN Function and Its Relation to Performance in Schizophrenia}

In the setting of reduced accuracy during SIRP performance, patients also exhibited reduced FPCN activation during error epochs, a finding that in itself resonates with previous reports describing FPCN dysfunction in schizophrenia. Further, during error epochs, the relationship between left dlPFC activation and overall accuracy was stronger in patients than controls. It remains unclear, though, whether diminished error-related FPCN activation in patients primarily reflects a failure of sustained engagement in task, or, alternatively, reduced baseline activity or impaired activation of error-monitoring circuitry. Although limited by substantial bin size discrepancies, a direct contrast of correct and error epochs (Supplementary Figure S3) suggests that controls-but not patients-scale up activation in right frontoparietal regions and bilateral dACC during errors. The $\mathrm{dACC}$ is a key node in the error-monitoring circuit that coordinates with FPCN (Agam et al, 2013) and shows hypoactivation in schizophrenia patients following antisaccade errors (Polli et al, 2008). As our task was not specifically designed to induce errors, other experimental designs may be more helpful in addressing this issue.

However, other findings suggest that FPCN function during WM in patients can be surprisingly robust. During correct epochs, patients' load-dependent activation within the task-positive network did not differ from that of controls, 
suggesting a capacity for normal FPCN activation. Indeed, several other investigators using cognitive control (Lesh et al, 2015) and WM (Johnson et al, 2006; Walter et al, 2007) tasks have also reported no significant difference in dlPFC activation during correct trials between medicated first-episode schizophrenia patients and healthy individuals. Further, in our study, although positive symptoms negatively correlated with activation in FPCN (echoing previous results from Menon et al, 2001), patients still showed normal activation of the network during correct trials. Finally, FPCN regions that showed reduced coupling during resting state in our study did not appear to differ in functional connectivity during task. Taken together, these data suggest that (1) patients are capable of relatively normal FPCN physiology during WM and (2) previously reported FPCN abnormalities in block-design fMRI studies principally reflect the influence of error epochs, which contribute to the block-averaged signal disproportionally in patients.

\section{Limbic and Default vmPFC and Their Relation to Performance in Schizophrenia}

Despite the clear role of FPCN in mediating WM processes (Curtis and D'Esposito, 2003; Heinzel et al, 2014), our findings call into question whether physiology differences outside of the FPCN are more fundamentally relevant to altered WM processing in schizophrenia. Arguably, such changes should not be confounded by performance differences between the groups. Like many executive function tasks, the SIRP deactivates limbic and default networks (Fryer et al, 2013). Regardless of performance, schizophrenia patients failed to deactivate bilateral vmPFC, a region assigned to the limbic network (Yeo et al, 2011). Patients also showed abnormally strong resting functional connectivity between vmPFC and IOFC, a region previously associated with emotion regulation processes and rumination (Eryilmaz et al, 2014; Goldin et al, 2008). Altered prefrontal connectivity in schizophrenia patients may underlie impaired filtering of irrelevant information (Anticevic et al, 2012), a phenomenon that may have contributed to the inverse correlation between vmPFC-1OFC connectivity and accuracy in our study.

Furthermore, inverse to the FPCN pattern, activity during error epochs in parts of vmPFC previously assigned to DMN (Yeo et al, 2011) correlated negatively with accuracy rates in the patient group. DMN deactivation has been linked to successful performance in cognitive tasks (Anticevic et al, 2010; Hahn et al, 2007), whereas failure of DMN deactivation during verbal WM has been observed in schizophrenia (Matsuo et al, 2013). Functional connectivity changes in DMN have also been reported in schizophrenia patients and their first-degree relatives (Garrity et al, 2007; Whitfield-Gabrieli et al, 2009). Effective connectivity between areas of the DMN including vmPFC has been correlated with WM performance in schizophrenia and bipolar patients (Wu et al, 2014). Taken together, our findings are consistent with the previous studies and suggest that task-related deactivation of the DMN during WM performance is associated with better performance in schizophrenia.

\section{Thalamus-FPCN Disconnection in Schizophrenia During Task Performance}

The thalamus, a critical node in thalamo-cortico-basal ganglia circuitry, also showed hypoactivation in patients during error epochs. Activation maps for 'Correct $v s$ fix' and 'Error $v s$ fix' contrasts (Supplementary Figure S2) suggests that for patients, correct performance depended strongly on activation of the thalamus. Interestingly, the consistent coactivation of thalamus and cortical FPCN regions in controls (regardless of performance) raises the question of whether the thalamus modulates cortical activity in the FPCN. Indeed, functional connectivity analysis of task blocks showed that the thalamus was more strongly coupled with regions of the FPCN such as dmPFC, dACC and left IPL in controls than in patients. As this thalamocortical connectivity did not differ between the groups during resting state, it is possible that the thalamus has a task-specific role in modulating FPCN activation, an idea supported by the observed correlation between left thalamus-dmPFC coupling and accuracy rate. Notably, in a mouse pharmacogenetic study, decreases in mediodorsal thalamic activity triggered selective impairments in spatial WM and disrupted synchrony between the thalamus and $\mathrm{mPFC}$, implying a causal relationship (Parnaudeau et al, 2013). Although we are limited by the resolution of our functional space, based on prior diffusion imaging-guided parcellation of the human thalamus (Behrens et al, 2003; Mang et al, 2012), we estimate that the peak difference between the groups in the thalamus lies in the medial anterior complex, which has extensive projections to the prefrontal cortex and encompasses the mediodorsal thalamus. Therefore, our results may concur with the direct evidence on thalamus-mPFC connectivity provided by Parnaudeau and colleagues.

Although the exact role of the thalamus in WM is unclear, evidence from primate studies suggests that mediodorsal nucleus of the thalamus might transmit prospective motor information to the dlPFC (Tanibuchi and Goldman-Rakic, 2003; Watanabe and Funahashi, 2012). A meta-analysis of human imaging studies reported the left thalamus, dlPFC, and dACC to be activated significantly less in schizophrenia patients during cognitive tasks (Minzenberg et al, 2009). In addition, structural thalamocortical connectivity to the lateral prefrontal cortex was previously associated with WM performance (Marenco et al, 2012). In addition to the decreased thalamus-FPCN coupling, we also observed abnormally increased connectivity between the thalamus and parahippocampal gyrus in patients during task performance. Structural changes in parahippocampal gyrus in patients with chronic schizophrenia have been previously reported (Schreiber et al, 2011). The current results therefore suggest that a shift from a normal thalamocortical (specifically, FPCN) coupling to an abnormal thalamolimbic coupling may contribute to WM differences between controls and patients.

One potential limitation of studies, including the present one, of chronic schizophrenia patients is the difficulty of controlling for the medication effects. Notably, despite equivalent performance compared with controls, unmedicated individuals with high genetic loading for schizophrenia exhibit abnormal FPCN activation (Seidman et al, 2006) and medial prefrontal deactivation (Landin-Romero et al, 2014) 
during WM. A recent multimodal study on first-episode psychosis also showed that medicated patients and healthy controls do not differ significantly in dlPFC activation during a cognitive control task (Lesh et al, 2015). It is therefore possible that the lack of difference in dlPFC activation between patients and controls during correct performance partially reflects the medication effects. Further, the small, but statistically significant difference in estimated verbal IQ between the groups may also contribute to differences in activation and connectivity, despite matching for other key demographic variables.

An additional limitation of our design was that it collapsed all error types into a single category to maximize statistical power. It is possible that controls and patients committed different type of errors (eg, honest mistakes or slips) and some of the group differences in activation may have arisen due to these differences. It is important to note, however, that our additional analysis in which we regressed out average reaction time values shows that our results were not confounded by reaction time differences between the groups. Finally, some degree of co-activation during task might have contributed to the task-based connectivity measure for certain pairs of regions.

In conclusion, using an approach that facilitated comparison of schizophrenia patients and controls despite performance differences, we demonstrated that patients exhibit robust load-dependent thalamic and FPCN activation during accurate WM performance, but show deficient FPCN activation during error epochs. However, patients fail to deactivate the medial prefrontal cortex regardless of performance. Connectivity results further substantiate that altered thalamic and limbic network functions underlie WM performance differences in schizophrenia patients, and underscore the importance of looking beyond frontoparietal network function in studies of WM abnormalities.

\section{FUNDING AND DISCLOSURE}

This work was supported by NIH (K23MH084059, R01MH101425, 1S10RR023043, and 1S10RR023401), HHMI (Physician-Scientist Early Career Award), and Pamlab. JLR has previously received grant support and consulting fees from Pamlab. The remaining authors declare no conflict of interest.

\section{ACKNOWLEDGMENTS}

We thank the study participants, and are grateful to Drs Daphne Holt and Isik Karahanoglu for helpful discussion relating to the manuscript.

\section{REFERENCES}

Agam Y, Carey C, Barton JJ, Dyckman KA, Lee AK, Vangel M et al (2013). Network dynamics underlying speed-accuracy trade-offs in response to errors. PloS ONE 8: e73692.

Anticevic A, Repovs G, Krystal JH, Barch DM (2012). A broken filter: prefrontal functional connectivity abnormalities in schizophrenia during working memory interference. Schizophr Res 141: 8-14.

Anticevic A, Repovs G, Shulman GL, Barch DM (2010). When less is more: TPJ and default network deactivation during encoding predicts working memory performance. Neuroimage 49: 2638-2648.

Baker JT, Holmes AJ, Masters GA, Yeo BT, Krienen F, Buckner RL et al (2014). Disruption of cortical association networks in schizophrenia and psychotic bipolar disorder. JAMA Psychiatry 71: 109-118.

Behrens TE, Johansen-Berg H, Woolrich MW, Smith SM, WheelerKingshott CA, Boulby PA et al (2003). Non-invasive mapping of connections between human thalamus and cortex using diffusion imaging. Nat Neurosci 6: 750-757.

Buckner RL (2010). Human functional connectivity: new tools, unresolved questions. Proc Natl Acad Sci USA 107: 10769-10770.

Burock MA, Dale AM (2000). Estimation and detection of eventrelated fMRI signals with temporally correlated noise: a statistically efficient and unbiased approach. Hum Brain Mapp 11: 249-260.

Carter CS, Perlstein W, Ganguli R, Brar J, Mintun M, Cohen JD (1998). Functional hypofrontality and working memory dysfunction in schizophrenia. Am J Psychiatry 155: 1285-1287.

Chai XJ, Castanon AN, Ongur D, Whitfield-Gabrieli S (2012). Anticorrelations in resting state networks without global signal regression. Neuroimage 59: 1420-1428.

Curtis CE, D'Esposito M (2003). Persistent activity in the prefrontal cortex during working memory. Trends Cognit Sci 7: 415-423.

D'Esposito M (2007). From cognitive to neural models of working memory. Philos Trans $R$ Soc Lond B Biol Sci 362: 761-772.

Dale AM, Buckner RL (1997). Selective averaging of rapidly presented individual trials using fMRI. Hum Brain Mapp 5: 329-340.

Eryilmaz H, Van De Ville D, Schwartz S, Vuilleumier P (2014). Lasting impact of regret and gratification on resting brain activity and its relation to depressive traits. J Neurosci 34: 7825-7835.

Fryer SL, Woods SW, Kiehl KA, Calhoun VD, Pearlson GD, Roach BJ et al (2013). Deficient suppression of default mode regions during working memory in individuals with early psychosis and at clinical high-risk for psychosis. Front Psychiatry 4: 92.

Garrity AG, Pearlson GD, McKiernan K, Lloyd D, Kiehl KA, Calhoun VD (2007). Aberrant "default mode" functional connectivity in schizophrenia. Am J Psychiatry 164: 450-457.

Goldin PR, McRae K, Ramel W, Gross JJ (2008). The neural bases of emotion regulation: reappraisal and suppression of negative emotion. Biol Psychiatry 63: 577-586.

Hagler DJ Jr, Saygin AP, Sereno MI (2006). Smoothing and cluster thresholding for cortical surface-based group analysis of fMRI data. Neuroimage 33: 1093-1103.

Hahn B, Ross TJ, Yang Y, Kim I, Huestis MA, Stein EA (2007). Nicotine enhances visuospatial attention by deactivating areas of the resting brain default network. J Neurosci 27: 3477-3489.

Heinzel S, Lorenz RC, Brockhaus WR, Wustenberg T, Kathmann N, Heinz A et al (2014). Working memory load-dependent brain response predicts behavioral training gains in older adults. J Neurosci 34: 1224-1233.

Jansma JM, Ramsey NF, van der Wee NJ, Kahn RS (2004). Working memory capacity in schizophrenia: a parametric fMRI study. Schizophr Res 68: 159-171.

Johnson MR, Morris NA, Astur RS, Calhoun VD, Mathalon DH, Kiehl KA et al (2006). A functional magnetic resonance imaging study of working memory abnormalities in schizophrenia. Biol Psychiatry 60: 11-21.

Karlsgodt KH, Glahn DC, van Erp TG, Therman S, Huttunen M, Manninen $\mathrm{M}$ et al (2007). The relationship between performance and fMRI signal during working memory in patients with schizophrenia, unaffected co-twins, and control subjects. Schizophr Res 89: 191-197.

Kim DI, Manoach DS, Mathalon DH, Turner JA, Mannell M, Brown GG et al (2009). Dysregulation of working memory and default-mode networks in schizophrenia using independent 
component analysis, an fBIRN and MCIC study. Hum Brain Mapp 30: 3795-3811.

Landin-Romero R, McKenna PJ, Salgado-Pineda P, Sarro S, Aguirre C, Sarri C et al (2014). Failure of deactivation in the default mode network: a trait marker for schizophrenia? Psychol Med 45: 1315-1325.

Lesh TA, Tanase C, Geib BR, Niendam TA, Yoon JH, Minzenberg MJ et al (2015). A multimodal analysis of antipsychotic effects on brain structure and function in first-episode Schizophrenia. JAMA Psychiatry 72: 226-234.

Mang SC, Busza A, Reiterer S, Grodd W, Klose AU (2012). Thalamus segmentation based on the local diffusion direction: a group study. Magn Reson Med 67: 118-126.

Manoach DS (2003). Prefrontal cortex dysfunction during working memory performance in schizophrenia: reconciling discrepant findings. Schizophr Res 60: 285-298.

Manoach DS, Gollub RL, Benson ES, Searl MM, Goff DC, Halpern E et al (2000). Schizophrenic subjects show aberrant fMRI activation of dorsolateral prefrontal cortex and basal ganglia during working memory performance. Biol Psychiatry 48: 99-109.

Manoach DS, Press DZ, Thangaraj V, Searl MM, Goff DC, Halpern E et al (1999). Schizophrenic subjects activate dorsolateral prefrontal cortex during a working memory task, as measured by fMRI. Biol Psychiatry 45: 1128-1137.

Marenco S, Stein JL, Savostyanova AA, Sambataro F, Tan HY, Goldman AL et al (2012). Investigation of anatomical thalamocortical connectivity and FMRI activation in schizophrenia. Neuropsychopharmacology 37: 499-507.

Matsuo K, Chen SH, Liu CM, Liu CC, Hwang TJ, Hsieh MH et al (2013). Stable signatures of schizophrenia in the corticalsubcortical-cerebellar network using fMRI of verbal working memory. Schizophr Res 151: 133-140.

Meda SA, Stevens MC, Folley BS, Calhoun VD, Pearlson GD (2009). Evidence for anomalous network connectivity during working memory encoding in schizophrenia: an ICA based analysis. Plos ONE 4: e7911.

Menon V, Anagnoson RT, Mathalon DH, Glover GH, Pfefferbaum A (2001). Functional neuroanatomy of auditory working memory in schizophrenia: relation to positive and negative symptoms. Neuroimage 13: 433-446.

Minzenberg MJ, Laird AR, Thelen S, Carter CS, Glahn DC (2009). Meta-analysis of 41 functional neuroimaging studies of executive function in schizophrenia. Arch Gen Psychiatry 66: 811-822.

Parnaudeau S, O'Neill PK, Bolkan SS, Ward RD, Abbas AI, Roth BL et al (2013). Inhibition of mediodorsal thalamus disrupts thalamofrontal connectivity and cognition. Neuron 77: 1151-1162.

Polli FE, Barton JJ, Thakkar KN, Greve DN, Goff DC, Rauch SL et al (2008). Reduced error-related activation in two anterior cingulate circuits is related to impaired performance in schizophrenia. Brain 131(Pt 4): 971-986.

Potkin SG, Turner JA, Brown GG, McCarthy G, Greve DN, Glover GH et al (2009). Working memory and DLPFC inefficiency in schizophrenia: the FBIRN study. Schizophr Bull 35: 19-31.

Power JD, Barnes KA, Snyder AZ, Schlaggar BL, Petersen SE (2012). Spurious but systematic correlations in functional connectivity MRI networks arise from subject motion. NeuroImage 59: 2142-2154.

Scholvinck ML, Maier A, Ye FQ, Duyn JH, Leopold DA (2010). Neural basis of global resting-state fMRI activity. Proc Natl Acad Sci USA 107: 10238-10243.

Schreiber S, Bernstein HG, Fendrich R, Stauch R, Ketzler B, Dobrowolny $\mathrm{H}$ et al (2011). Increased density of GAD65/67 immunoreactive neurons in the posterior subiculum and parahippocampal gyrus in treated patients with chronic schizophrenia. World J Biol Psychiatry 12: 57-65.

Seidman LJ, Thermenos HW, Poldrack RA, Peace NK, Koch JK, Faraone SV et al (2006). Altered brain activation in dorsolateral prefrontal cortex in adolescents and young adults at genetic risk for schizophrenia: an fMRI study of working memory. Schizophr Res 85: 58-72.

Sternberg S (1966). High-speed scanning in human memory. Science 153: 652-654.

Tanibuchi I, Goldman-Rakic PS (2003). Dissociation of spatial-, object-, and sound-coding neurons in the mediodorsal nucleus of the primate thalamus. J Neurophysiol 89: 1067-1077.

Van Snellenberg JX, Torres IJ, Thornton AE (2006). Functional neuroimaging of working memory in schizophrenia: task performance as a moderating variable. Neuropsychology 20: 497-510.

Walter H, Vasic N, Hose A, Spitzer M, Wolf RC (2007). Working memory dysfunction in schizophrenia compared to healthy controls and patients with depression: evidence from eventrelated fMRI. Neuroimage 35: 1551-1561.

Watanabe Y, Funahashi S (2012). Thalamic mediodorsal nucleus and working memory. Neurosci Biobehav Rev 36: 134-142.

Whitfield-Gabrieli S, Thermenos HW, Milanovic S, Tsuang MT, Faraone SV, McCarley RW et al (2009). Hyperactivity and hyperconnectivity of the default network in schizophrenia and in first-degree relatives of persons with schizophrenia. Proc Natl Acad Sci USA 106: 1279-1284.

Wu G, Wang Y, Mwansisya TE, Pu W, Zhang H, Liu C et al (2014). Effective connectivity of the posterior cingulate and medial prefrontal cortices relates to working memory impairment in schizophrenic and bipolar patients. Schizophr Res 158: 85-90.

Yendiki A, Greve DN, Wallace S, Vangel M, Bockholt J, Mueller BA et al (2010). Multi-site characterization of an fMRI working memory paradigm: reliability of activation indices. Neuroimage 53: 119-131.

Yeo BT, Krienen FM, Sepulcre J, Sabuncu MR, Lashkari D, Hollinshead $\mathrm{M}$ et al (2011). The organization of the human cerebral cortex estimated by intrinsic functional connectivity. J Neurophysiol 106: 1125-1165.

Supplementary Information accompanies the paper on the Neuropsychopharmacology website (http://www.nature.com/npp) 